\title{
Methane monitoring by near infrared photoacoustic spectroscopy: The importance of relaxation phenomena
}

\author{
S. Schilt, J.-Ph. Besson and L. Thévenaz \\ Nanophotonics and Metrology Laboratory, Swiss Federal Institute of Technology, \\ 1015 Lausanne, Switzerland, e-mail: stephane.schilt@epfl.ch
}

\begin{abstract}
The importance of molecular relaxation in photoacoustic spectroscopy is discussed. The particular case of methane monitoring in dry oxygen using a $1.65-\mu \mathrm{m}$ laser-based photoacoustic sensor is reported. The slow vibration-to-translation energy transfer occurring in this gas mixture results in a drastic reduction of the detection sensitivity. A quadratic response of the sensor to the methane concentration is also reported and explained by molecular relaxation effects.
\end{abstract}

\section{INFLUENCE OF MOLECULAR RELAXATION IN PHOTOACOUSTICS}

Photoacoustic (PA) spectroscopy basically consists in exciting an absorbing gas sample with a modulated laser beam at proper wavelength and detecting the generated acoustic wave using a microphone. The acoustic wave results from molecular absorption of photons and subsequent deactivation of the excited vibrational state via inelastic collisions with the nearby molecules (collisional relaxation). When the excited vibrational energy is transferred into kinetic energy of the surrounding molecules, a periodic heating occurs in the sample and induces the pressure wave. In order to efficiently generate a PA signal, the typical timescale of the vibration-to-translation (V-T) energy transfer should be much shorter than the period of the laser modulation (generally in the millisecond range). This condition is most often fulfilled, as the collisional relaxation time of most molecules ranges from nanosecond to hundreds of microseconds at atmospheric pressure. However, the relaxation time of some particular molecules may be orders of magnitude longer. In particular, this is the case for a few diatomic molecules, such as $\mathrm{N}_{2}$ or $\mathrm{O}_{2}$ (see Table 1), and for collisions with some particular partners $\left(\mathrm{N}_{2}\right.$ and $\mathrm{O}_{2}$, too). When the timescale of the $\mathrm{V}$-T processes is comparable or larger than the modulation period, the PA signal is poorly excited.

Table 1. Examples of relaxation rates of some vibrational states with different collisional partners. Reactions labelled in bold correspond to $\mathrm{V}-\mathrm{T}$ processes, the others to $\mathrm{V}-\mathrm{V}$ processes.

\begin{tabular}{lccccc}
\hline \multicolumn{1}{c}{ Reaction } & $\begin{array}{c}\text { Rate } \\
{\left[\mathrm{s}^{-1} \mathrm{~atm}^{-1}\right]}\end{array}$ & Ref. & Reaction & $\begin{array}{c}\text { Rate } \\
{\left[\mathrm{s}^{-1} \mathrm{~atm}^{-1}\right]}\end{array}$ & Ref. \\
\hline$\left(\boldsymbol{R}_{1}\right) \mathrm{CH}_{4}^{*}\left(n v_{4}\right)+\mathrm{CH}_{4} \rightarrow \mathrm{CH}_{4}^{*}\left((n-1) v_{4}\right)+\mathrm{CH}_{4}^{*}\left(v_{4}\right)$ & $2.7 \cdot 10^{8}$ & {$[1]$} & $\left(\boldsymbol{R}_{7}\right) \mathrm{O}_{2}^{*}(v)+\mathrm{O}_{2} \rightarrow \mathrm{O}_{2}+\mathrm{O}_{2}$ & $6.3 \cdot 10$ & {$[3]$} \\
$\left(\boldsymbol{R}_{\mathbf{2}}\right) \mathrm{CH}_{4}^{*}\left(n v_{4}\right)+\mathrm{CH}_{4} \rightarrow \mathrm{CH}_{4}^{*}\left((n-1) v_{4}\right)+\mathrm{CH}_{4}$ & $8 \cdot 10^{5}$ & {$[1]$} & $\left(\boldsymbol{R}_{\mathbf{8}}\right) \mathrm{O}_{2}^{*}(v)+\mathrm{N}_{2} \rightarrow \mathrm{O}_{2}+\mathrm{N}_{2}$ & $4 \cdot 10$ & {$[3]$} \\
$\left(R_{3}\right) \mathrm{CH}_{4}^{*}\left(n v_{4}\right)+\mathrm{O}_{2} \rightarrow \mathrm{CH}_{4}^{*}\left((n-1) v_{4}\right)+\mathrm{O}_{2}^{*}(v)$ & $\sim 3 \cdot 10^{7}$ & $\left(\boldsymbol{R}_{\mathbf{9}}\right) \mathrm{O}_{2}^{*}(v)+\mathrm{H}_{2} \mathrm{O} \rightarrow \mathrm{O}_{2}+\mathrm{H}_{2} \mathrm{O}$ & $1.1 \cdot 10^{6}$ & {$[3]$} \\
$\left(\boldsymbol{R}_{\mathbf{4}}\right) \mathrm{CH}_{4}^{*}\left(n v_{4}\right)+\mathrm{O}_{2} \rightarrow \mathrm{CH}_{4}^{*}\left((n-1) v_{4}\right)+\mathrm{O}_{2}$ & $1.3 \cdot 10^{5}$ & {$[2]$} & $\left(R_{10}\right) \mathrm{O}_{2}^{*}(v)+\mathrm{CH}_{4} \rightarrow \mathrm{O}_{2}+\mathrm{CH}_{4}^{*}\left(v_{4}\right)$ & $2 \cdot 10^{7}$ & {$[4]$} \\
$\left(\boldsymbol{R}_{\mathbf{5}}\right) \mathrm{CH}_{4}^{*}\left(n v_{4}\right)+\mathrm{N}_{2} \rightarrow \mathrm{CH}_{4}^{*}\left((n-1) v_{4}\right)+\mathrm{N}_{2}$ & $8 \cdot 10^{4}$ & {$[1]$} & $\left(\boldsymbol{R}_{\mathbf{1 1}}\right) \mathrm{O}_{2}^{*}(v)+\mathrm{CH}_{4} \rightarrow \mathrm{O}_{2}+\mathrm{CH}_{4}$ & $1 \cdot 10^{6}$ & {$[5]$} \\
$\left(\boldsymbol{R}_{\mathbf{6}}\right) \mathrm{N}_{2}^{*}(v)+\mathrm{N}_{2} \rightarrow \mathrm{N}_{2}+\mathrm{N}_{2}$ & 1.0 & {$[3]$} & $\left(\boldsymbol{R}_{\mathbf{1 2}}\right) \mathrm{O}_{2}^{*}(v)+\mathrm{He} \rightarrow \mathrm{O}_{2}+\mathrm{He}$ & $2.3 \cdot 10^{4}$ & {$[6]$} \\
\hline
\end{tabular}




\section{EXPERIMENTS}

A fibre-coupled PA sensor based on a $1.65-\mu \mathrm{m}$ DFB laser and a resonant PA cell operated on its first longitudinal resonance around $1 \mathrm{kHz}$ has been developed for $\mathrm{CH}_{4}$ detection [7]. Methane has been measured in different buffer gases using this set-up, in order to emphasize the importance of molecular relaxation processes. Whereas a sub-ppm detection limit has been achieved for $\mathrm{CH}_{4}$ in dry nitrogen, the performances are reduced by more than one order of magnitude when $\mathrm{CH}_{4}$ is measured in pure oxygen, or even in dry $\mathrm{N}_{2}-\mathrm{O}_{2}$ mixtures containing more than $10 \%$ of $\mathrm{O}_{2}$, as shown in Figure 1 . Furthermore, the PA signal measured in $\mathrm{O}_{2}$ does not present the usual linear dependence on the $\mathrm{CH}_{4}$ concentration, but shows a quadratic variation, as illustrated in Figure 2. Finally, when a few percent of helium is added to $\mathrm{O}_{2}$, the $\mathrm{CH}_{4}$ PA signal retrieves the usual linear dependence on the gas concentration and the detection sensitivity becomes comparable to the value obtained for $\mathrm{CH}_{4}$ in $\mathrm{N}_{2}$. All these observed phenomena are related to molecular relaxation effects. They can be explained by the deactivation steps of the $\mathrm{CH}_{4}$ excited state and the relaxation rates corresponding to collisions with the different types of surrounding molecules.

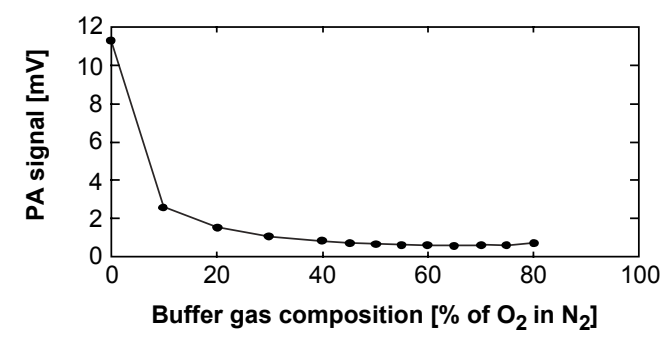

Figure 1. Amplitude of the $\mathrm{PA}$ signal produced by $100 \mathrm{ppm}$ of $\mathrm{CH}_{4}$ in a $\mathrm{N}_{2}-\mathrm{O}_{2}$ mixture.

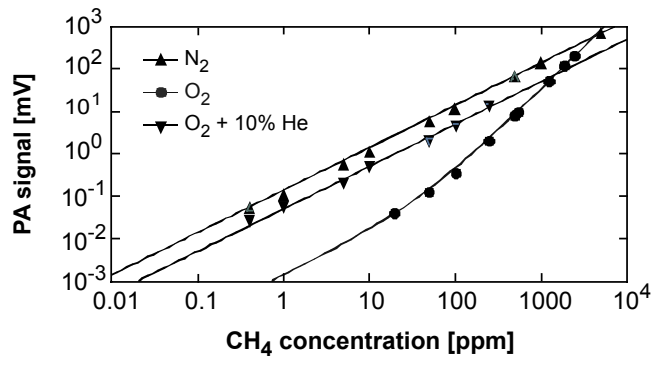

Figure 2. PA signal measured as a function of the $\mathrm{CH}_{4}$ concentration in different buffer gases. Circles are experimental data and the lines represent a linear fit $(y$ $=a x$ ) for $\mathrm{N}_{2}$ and $\mathrm{O}_{2}-\mathrm{He}$ mixture, and a second-order polynomial fit $\left(y=a x^{2}+b x\right)$ for $\mathrm{O}_{2}$.

\section{DISCUSSION}

\section{1 $\mathrm{CH}_{4}$ relaxation in $\mathrm{O}_{2}$}

The $\mathrm{CH}_{4}$ molecule has four vibrational modes: two bending vibrations $v_{2}$ (asymmetric) and $v_{4}$ (symmetric), and two stretching vibrations $v_{1}$ (symmetric) and $v_{3}$ (asymmetric). The first excited bending levels $v_{2}$ and $v_{4}$ are located at 1533 and $1311 \mathrm{~cm}^{-1}$, respectively, and the stretching levels $v_{1}$ and $v_{3}$ at 2917 and $3019 \mathrm{~cm}^{-1}$, respectively, approximately two times higher than the two former ones. Consequently, the vibrational energies of methane appear as clusters (called polyads) of states interacting together through Fermi or Coriolis resonance. Due to the strong interactions existing between the states in a given polyad, the spectroscopic analysis of any vibrational state needs to take into account simultaneously all the states of the polyad to which the level belongs [2]. This characteristic is important for the understanding of the collisional relaxation of methane. In particular, very fast energy transfers (in the nanosecond range) occur between the states of the same polyad (intermodal transfer) for both $\mathrm{CH}_{4}-\mathrm{CH}_{4}$ and $\mathrm{CH}_{4}-\mathrm{O}_{2}$ collisions. The energy transfer between two polyads essentially occurs via the exchange of one $v_{4}$ vibrational quantum (see reactions $R_{1}$ and $R_{3}$ in Table 1). For $\mathrm{CH}_{4}-\mathrm{O}_{2}$ collisions, the de-excitation of the $\mathrm{CH}_{4}$ molecule is accompanied by a resonant excitation of the first vibrational state of oxygen, $\mathrm{O}_{2}{ }^{*}(\mathrm{v})$, due to its close matching with the level 
$\mathrm{CH}_{4}{ }^{*}\left(v_{4}\right)$. For weak $\mathrm{CH}_{4}$ concentrations, the transfer to the lower polyads occurs only via the resonant process $R_{3}$. Therefore, the whole $1.65-\mu \mathrm{m}$ laser energy initially absorbed in the $\mathrm{CH}_{4}{ }^{*}\left(2 v_{3}\right)$ state is eventually stored in the $\mathrm{O}_{2}{ }^{*}(v)$ vibrational state. According to Table 1 (see reaction $R_{7}$ ), this state has a relaxation rate that is much smaller than the laser modulation frequency $(1 \mathrm{kHz})$, so that no PA signal is coherently generated with the laser modulation. This explains the very small PA signal observed when measuring $\mathrm{CH}_{4}$ in $\mathrm{O}_{2}$. The situation is totally different for $\mathrm{CH}_{4}$ in $\mathrm{N}_{2}$, as the first vibrational state of $\mathrm{N}_{2}$ has a larger energy than $\mathrm{CH}_{4}{ }^{*}\left(v_{4}\right)$ level, so that no $\mathrm{V}-\mathrm{V}$ coupling can occur.

\subsection{Quadratic dependence of the $\mathrm{PA}$ signal to $\mathrm{CH}_{4}$ concentration}

Whereas the collisional V-T relaxation of $\mathrm{O}_{2}{ }^{*}(\mathrm{v})$ is very slow for $\mathrm{O}_{2}-\mathrm{O}_{2}$ collisions, it is more than four orders of magnitude faster for $\mathrm{O}_{2}-\mathrm{CH}_{4}$ collisions (see reaction $R_{11}$ in Table 1). So even for small $\mathrm{CH}_{4}$ concentrations, the relaxation time of $\mathrm{O}_{2}{ }^{*}(v)$ state is reduced when increasing the $\mathrm{CH}_{4}$ concentration. The relaxation rate of $\mathrm{O}_{2}{ }^{*}(v)$ state in a $\mathrm{O}_{2}-M$ mixture changes with the concentration $C_{M}$ of the collisional partner $M$ according to:

$$
\tau_{\mathrm{O}_{2}}{ }^{-1}=\left(1-C_{M}\right) \tau_{\mathrm{O}_{2}-\mathrm{O}_{2}}{ }^{-1}+C_{M} \tau_{\mathrm{O}_{2}-M}{ }^{-1} .
$$

For small $\mathrm{CH}_{4}$ concentrations, a linear variation of the relaxation rate is obtained $(y=a x+b)$, as shown in Figure 3. This produces a linear increase of the PA signal, as the ratio of the relaxation time to the modulation period is reduced. This linear

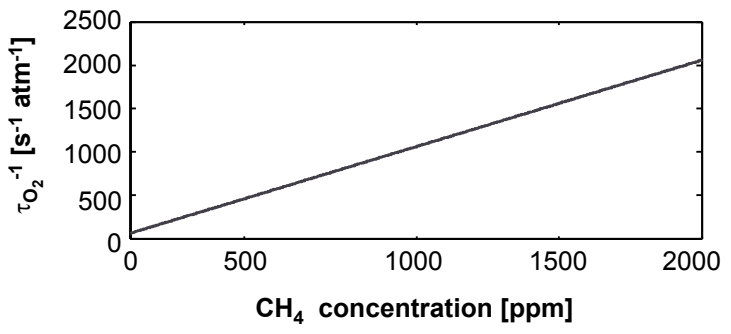

Figure 3. Variation of the V-T relaxation time in a $\mathrm{O}_{2}-\mathrm{CH}_{4}$ mixture.

dependence of the PA signal only results from the relaxation process. In addition to it, the PA signal also presents the usual linear variation on the gas concentration $(y=a x)$, due to the increasing amount of energy absorbed in the sample. Therefore, a double linear dependence of the PA signal to the $\mathrm{CH}_{4}$ concentration occurs, which results in the quadratic variation experimentally observed $\left(y=a x^{2}+b x\right)$.

\subsection{Effect of the presence of helium in the buffer gas}

As methane, helium is an efficient collisional partner for the vibrational relaxation of $\mathrm{O}_{2}{ }^{*}(v)$ (see reaction $R_{12}$ in Table 1$)$. In a $\mathrm{O}_{2}-\mathrm{He}$ mixture, the $\mathrm{O}_{2}{ }^{*}(v)$ relaxation rate changes with helium concentration according to Eq. (1). With the addition of $10 \%$ of helium, the $\mathrm{O}_{2}{ }^{*}(\mathrm{v})$ relaxation time decreases from $20 \mathrm{~ms}$ to $0.4 \mathrm{~ms}$, which is smaller than the laser modulation period. Consequently, the absorbed laser energy fully contributes to the generation of the PA signal and the usual behaviour is again observed (linear variation of the PA signal with the $\mathrm{CH}_{4}$ concentration).

\section{CONCLUSION}

The importance of collisional relaxation processes in the PA signal generation has been demonstrated in the particular case of $\mathrm{CH}_{4}$ detection in dry $\mathrm{O}_{2}$. A resonant coupling between $\mathrm{CH}_{4}{ }^{*}\left(v_{4}\right)$ and $\mathrm{O}_{2}{ }^{*}(v)$ vibrational states associated to the small relaxation rate of the $\mathrm{O}_{2}{ }^{*}(v)$ excited state lead to an important loss of sensitivity in comparison to the case of $\mathrm{CH}_{4}$ in $\mathrm{N}_{2}$. A totally unusual quadratic variation of the PA signal with the $\mathrm{CH}_{4}$ concentration has also been observed and explained. Finally, a sensitive $\mathrm{CH}_{4}$ detection in dry $\mathrm{O}_{2}$ with a linear response has been demonstrated by the addition of a few percent of helium to the gas mixture, which accelerates the vibrational relaxation of the $\mathrm{O}_{2}{ }^{*}(v)$ excited state. 


\section{Acknowledgments}

The authors would like to acknowledge the Commission of Technology and Innovation (CTI) of the Swiss government and companies Omnisens SA and Daetwyler Fiber Optics SA for their financial support. The authors are also grateful to C. Boursier (Université Pierre et Marie Curie, Paris) for fruitful discussions.

\section{References}

[1] Boursier C., Ménard J., Doyennette L. and Menard-Bourcin F., J. Phys. Chem. A 107 (2003), 5280-5290.

[2] Doyennette L., Menard-Bourcin F., Ménard J., Boursier C. and Camy-Peyret C., J. Phys. Chem. A 102 (1998), 3849-3855.

[3] Bass H.E. and Bauer H.-J., Appl. Optics 12 (1973), 1506-1510.

[4] Yardley J.T. and Moore C.B., J. Chem. Phys. 48 (1968), 14-17.

[5] White D.L., J. Chem. Phys. 42 (1965), 2028-2032.

[6] White D.L. and Millikan R.C., J. Chem. Phys. 39 (1963), 1807-1808.

[7] Besson J.-Ph., Schilt S. and Thévenaz L., Spectrochim. Acta A 60 (2004), 3449-3456. 